\title{
Combined RASSF1A and RASSF2A Promoter Methylation Analysis as Diagnostic Biomarker for Bladder Cancer
}

\author{
Wei Meng, ${ }^{1}$ Alexander Huebner, ${ }^{1}$ Ahmad Shabsigh, ${ }^{2}$ \\ Arnab Chakravarti, ${ }^{1}$ and Tim Lautenschlaeger ${ }^{1}$ \\ ${ }^{1}$ Department of Radiation Oncology, The Ohio State University, Columbus, OH 43210, USA \\ ${ }^{2}$ Department of Urology, The Ohio State University, Columbus, OH 43210, USA \\ Correspondence should be addressed to Tim Lautenschlaeger, tl@osumc.edu
}

Received 1 November 2011; Accepted 29 December 2011

Academic Editor: Farida Latif

Copyright ( $) 2012$ Wei Meng et al. This is an open access article distributed under the Creative Commons Attribution License, which permits unrestricted use, distribution, and reproduction in any medium, provided the original work is properly cited.

\begin{abstract}
Promoter hypermethylation, a widely studied epigenetic event known to influence gene expression levels, has been proposed as a potential biomarker in multiple types of cancer. Clinical diagnostic biomarkers are needed for reliable prediction of bladder cancer recurrence. In this paper, DNA promoter methylation of five C-terminal Ras-association family members (RASSF1A, RASSF2A, RASSF4, RASSF5, and RASSF6) was studied in 64 formalin-fixed paraffin-embedded (FFPE) bladder cancer and normal adjacent tissues using methylation-specific high-resolution melting (MS-HRM) analysis. Results showed that 73\% (30/41) of transitional cell carcinoma, 100\% (3/3) of squamous cell carcinoma, and 100\% (4/4) of small cell carcinoma demonstrated promoter methylation of the RASSF1A or RASSF2A gene, but only $6 \%$ (1/16) of normal tissues had promoter methylation of RASSF genes. Testing positive for hypermethylation of RASSF1A or RASSF 2 A promoter provided $77 \%$ sensitivity and $94 \%$ specificity for identification of cancer tissues with an area under the curve of 0.854 , suggesting that promoter methylation analysis of RASSF1A and RASSF2A genes has potential for use as a recurrence biomarker for bladder cancer patients.
\end{abstract}

\section{Introduction}

In 2011, about 52,000 men and 17,000 women will be diagnosed with bladder cancer in the United States. Before a normal cell transforms into a bladder cancer cell, a series of molecular alterations are accumulated to initiate the process of transformation. Although we do not fully understand the mechanisms, DNA alterations including hypermethylation and somatic mutation are commonly observed events in human cancer. In a recent bladder cancer study FGFR3 mutation in combination with APC, RASSF1A, and SFRP2 methylation markers provided a sensitivity of $90 \%$ using tissue samples and $62 \%$ using paired urine samples to identify the presence of cancer with $100 \%$ specificity [1]. In nonsmall cell lung cancer (NSCLC) and breast cancer, studies showed that RASSF1A had different frequencies of methylation depending on histology $[2,3]$. In nasopharyngeal carcinoma, RASSF2A was frequently inactivated by its promoter methylation and the methylation correlated with lymph node metastasis [4]. The Ras-association family, also called RASSF tumor suppressor genes, currently includes 10 members. All of the RASSF proteins contain a Ras-association domain on their C-terminus (RASSF 1-6) or N-terminus (RASSF 710). Two important issues that are not previously addressed by studies of RASSF gene methylation are (1) whether all of the RASSF family members show aberrant methylation in bladder cancer and (2) whether methylation pattern of $R A S S F$ genes can be used as a diagnostic biomarker.

RASSF1A (Ras-association domain family 1 isoform A) is the first identified RASSF family member which is frequently epigenetically inactivated in a wide range of cancer types. As a tumor suppressor gene, $R A S S F 1 A$ regulates the activation of cell death [5], cell cycle [6], and microtubule formation [7]. The methylation signature of RASSF1A is thought to be among the earliest cellular changes in tumorigenesis [8]. As a potential tumor suppressor, RASSF2 plays a role in apoptosis and cell cycle arrest and is frequently downregulated in lung tumor cell lines by hypermethylation [9]. Although the $5^{\prime}$ CpG island of RASSF3 has been identified earlier, RASSF3 does not show methylation in glioma tumor cell lines [10]. 
RASSF4 is broadly expressed in different human tissues, but its expression is down-regulated by promoter hypermethylation in a majority of tumor cell lines and primary tumors [11]. As a proapoptotic Ras effector, RASSF5 (NORE1A) is frequently inactivated by promoter methylation in human tumors like glioma tumor cell lines, colorectal tumors, and lung cancer [12-15]. RASSF6 promotes apoptosis by cooperating with activated $K$-Ras to induce cell death and inhibit the tumor cell survival [16]. A high frequency of RASSF6 methylation is present in leukaemia-related diseases [17]. It appears that all of C-terminal RASSF family members have hypermethylation-induced gene inactivation in various types of cancer. While there is extensive literature on RASSF1A, other RASSF family members have not been studied as widely.

High-resolution melting (HRM) analysis is a new methodology that monitors the melting behavior of PCR amplicons by using DNA intercalating fluorescent dye [18]. Originally the LCGreen was used to develop a closed-tube method for genotyping and mutation scanning [19]. New high sensitive dyes such as EVA Green and SYTO 9 can be used at saturation concentration to monitor the denaturing process of PCR amplicons. Compared to traditional methylation specific PCR (MSP) method, HRM is a reliable and simple method for DNA methylation detection [20, 21].

In this study, to examine diagnostic value of RASSF gene methylation, we identified the methylation status of $\mathrm{CpG}$ islands associated with C-terminal RASSF $1-6$ in a group of formalin fixed paraffin embedded bladder cancer samples using a methylation specific HRM assay.

\section{Methods}

2.1. Control and FFPE Tumor Samples. Universal methylated and unmethylated DNA samples (Zymo Research Corp, orange, CA) were used as $100 \%$ and $0 \%$ methylated control. The methylated DNA was serially diluted in unmethylated DNA to create standard dilutions of $0 \%, 10 \%, 50 \%$, and $100 \%$ methylated DNA. The standard dilutions from $100 \%$ to $0 \%$ were used to semiquantitatively measure promoter methylation status of C-terminal RASSF genes in FFPE samples.

FFPE blocks from 48 bladder cancer patients were collected by the department of pathology and the Human Tissue Resource Network at The Ohio State University. The study was conducted in accordance with the Institutional Review Board guidelines. We obtained 16 paired tumor and matched normal adjacent tissues and 32 tumor tissues $(35$ males and 13 females, male-to-female ratio 2.7:1; median age 67 years, range $28-90$ years). Among these patients, 41 cases were diagnosed with transitional cell carcinoma; 4 cases were small cell carcinoma and 3 cases were squamous cell carcinoma. Clinicopathologic and demographic characteristic of bladder cancer samples are shown in Table 1.

2.2. DNA Extraction. DNA samples were extracted using Recover All Total Nucleic Acid Isolation Kit (Life Technologies Corporation, Carlsbad, CA). Briefly, 5-10 mg samples were sliced from paraffin blocks and deparaffinizated by xylene at $50^{\circ} \mathrm{C}$, followed by $100 \%$ ethanol wash. The air-dry tissue samples were digested by proteinase $\mathrm{K}$ for $24 \mathrm{hrs}$ in a microtube shaking incubator set at $50^{\circ} \mathrm{C}$. The digested samples were mixed with appropriate volume of isolation additive and $100 \%$ ethanol. After passing the mixture through the filter cartridge, the DNA and RNA were retained on the filter. The RNA was removed by on-filter RNase digestion. The DNA was purified by washing buffer and eluted with $95^{\circ} \mathrm{C}$ nuclease-free water.

2.3. Bisulfite Modification. The FFPE DNA bisulfite modification was processed using EZ DNA Methylation Kit (Zymo Research Corp, Orange, CA). The double-stranded DNA was denatured in M-Dilution Buffer for 15 minutes at $37^{\circ} \mathrm{C}$, and then CT Conversion Reagent was added to each sample. The samples were incubated in the dark at $50^{\circ} \mathrm{C}$ for 12 hours followed by $4^{\circ} \mathrm{C}$ for 10 minutes. After mixing with $\mathrm{M}$ Binding Buffer, the samples were passed through a ZymoSpin IC Column. The DNA purification and desulphonation were performed on the column. Finally, the bisulfitemodified DNA was eluted by M-Elution Buffer from column matrix.

2.4. MS-HRM Primer Design. MS-HRM is based on PCR amplification of bisulfite modified genomic DNA with subsequent HRM analysis of PCR amplicons. The primers were designed to amplify both methylated and unmethylated DNA. Because of DNA degradation in FFPE samples, the sizes of amplicons were limited to $80-180 \mathrm{bp}$. The free online tool from MethPrimer (http://www.urogene.org/methprimer/indexl.html) was used specifically for primer design in this RASSF promoter methylation study. Primer sequences and amplicon lengths are shown in Table 2.

2.5. High-Resolution Melting Analysis (HRM). PCR amplification and high-resolution melting analysis were carried out sequentially on a CFX96 real-time PCR system (Biorad, Hercules, CA). PCR was performed in a $20 \mu \mathrm{L}$ total volume containing: $10 \mu \mathrm{L} 2 \mathrm{X}$ Type-it HRM PCR Master Mix (QIAGEN, Hilden, Germany), $1 \mu \mathrm{L} 10 \mathrm{picomol} / \mu \mathrm{L}$ MSHRM primer, $8 \mu \mathrm{L}$ nuclease-free water, and $1 \mu \mathrm{L}$ bisulfite converted DNA (theoretical concentration $10 \mathrm{ng} / \mu \mathrm{L}$ ). The amplification consisted of $10 \mathrm{~min}$ at $95^{\circ} \mathrm{C}$, followed by 40 cycles of $10 \mathrm{~s}$ at $95^{\circ} \mathrm{C}, 30 \mathrm{~s}$ at annealing temperature and $10 \mathrm{~s}$ at $72^{\circ} \mathrm{C}$. High-resolution melting analysis were performed at the temperature ramping from $70-95^{\circ} \mathrm{C}$ by $0.2^{\circ} \mathrm{C} / \mathrm{s}$ and florescence acquisition was set per manufacturer's recommendation.

2.6. Statistical Analysis. Methylation was classified as positive if at least $10 \%$ methylation was measured. The chi-square test was used to examine the significant differences of methylation depending on histology and staging $(P \leq 0.05)$. To evaluate sensitivity and specificity of methylation as a predictive marker receiver operating characteristic (ROC) analysis was used. 
TABLE 1: Patient demographic information.

\begin{tabular}{|c|c|c|c|}
\hline & Study populations & & \\
\hline & Total $(N=64)$ & $\begin{array}{l}\text { Bladder cancer tissue } \\
\text { specimen }(n=48)\end{array}$ & $\begin{array}{c}\text { Matched bladder cancer and } \\
\text { normal adjacent tissues }(n=16)\end{array}$ \\
\hline Age (years): mean (range) & $68(28-90)$ & $67(28-90)$ & $69(53-90)$ \\
\hline Gender & & & \\
\hline Male & 35 & 35 & 12 \\
\hline Female & 13 & 13 & 4 \\
\hline Histologic cell type & & & \\
\hline Transitional cell carcinoma & 41 & 41 & 12 \\
\hline Small cell carcinoma & 4 & 4 & 1 \\
\hline Squamous cell carcinoma & 3 & 3 & 3 \\
\hline T stage & & & \\
\hline $\mathrm{T} 1$ & 9 & 9 & 2 \\
\hline $\mathrm{T} 2$ & 11 & 11 & 3 \\
\hline T3 & 18 & 18 & 8 \\
\hline $\mathrm{T} 4$ & 6 & 6 & 1 \\
\hline $\mathrm{Tx}$ & 4 & 4 & 4 \\
\hline $\mathrm{N}$ stage & & & \\
\hline N0 & 27 & 27 & 9 \\
\hline N1 & 8 & 8 & 2 \\
\hline $\mathrm{N} 2$ & 8 & 8 & 3 \\
\hline $\mathrm{Nx}$ & 5 & 5 & 2 \\
\hline
\end{tabular}

\section{Results and Discussion}

To generate a profile of C-terminal RASSF gene epigenetic changes in bladder cancer, 64 bladder FFPE tissue samples were examined by methylation-specific HRM assay. The C-terminal RASSF family members (RASSF1A, RASSF2A, RASSF4, RASSF5, and RASSF6) were analyzed in this study. In the UCSC Genome Browser, we found a CpG island in the RASSF3 promoter region. However, due to the dense CG dinucleotides on the CpG island of the identified RASSF3 promoter, no appropriate HRM primers could be designed for this gene.

3.1. Quality Assessment of Methylation-Specific HRM Assay. HRM assay uses double-stranded DNA binding dyes and requires less PCR optimization than other methods. The principle of HRM depends on recording the melting profile of double-stranded DNA samples. As double-stranded DNA is denatured, the fluorescence signal from dye bound to double-stranded DNA decreases. The melting profile is related to amplicon length, DNA sequence and GC content. The high-resolution melting requires smaller temperature increase steps $\left(<0.5^{\circ} \mathrm{C} / \mathrm{s}\right)$ between each fluorescence reading, which can provide detailed information of amplicon melting behavior.

MS-HRM is a semi-quantitative method for rapidly assessing the presence of DNA methylation. The standard curve of methylation was used to confirm the RASSF gene methylation. The bisulfite-modified fully methylated DNA was diluted in bisulfite-modified fully unmethylated DNA to obtain a series of methylation percentage: $0 \%, 10 \%$,

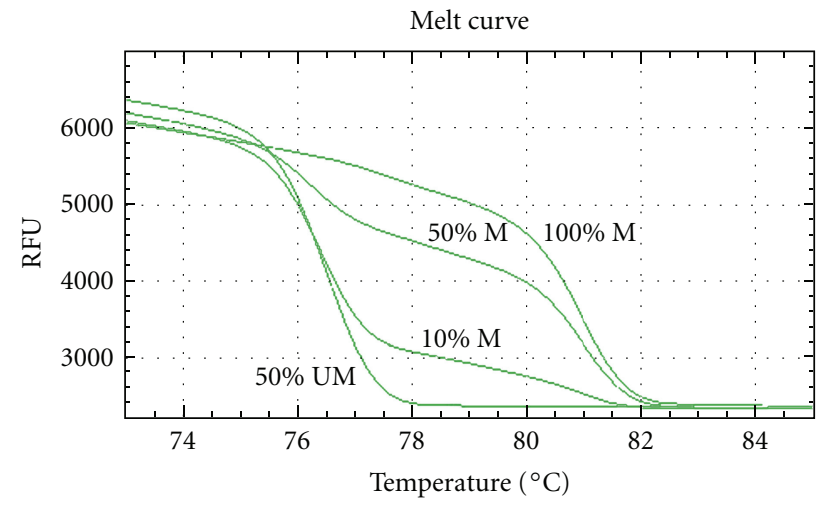

FIGURE 1: Standard curve constructed for RASSF promoter methylation. The dilutions of methylated DNA in unmethylated DNA are as follows: $0 \%$ methylation, $10 \%$ methylation, $50 \%$ methylation, and $100 \%$ methylation. Using Meth Primer software, a pair of primers was designed to amplify both methylated and unmethylated sequences after bisulfite conversion. The melting curves of $0 \%$ and $100 \%$ methylation indicate melting temperature of unmethylated sequence $\left(76.6^{\circ} \mathrm{C}\right)$ and methylated sequence $\left(81^{\circ} \mathrm{C}\right)$.

$50 \%$, and $100 \%$ methylation. Only samples containing more than $10 \%$ methylation were counted as methylated samples. The standard curve of RASSF2A methylation is shown in Figure 1.

3.2. RASSF Family Member Methylation Profile in Tumor and Normal Adjacent Tissue Samples. Promoter methylation was analyzed in tumor and normal adjacent tissues from 16 


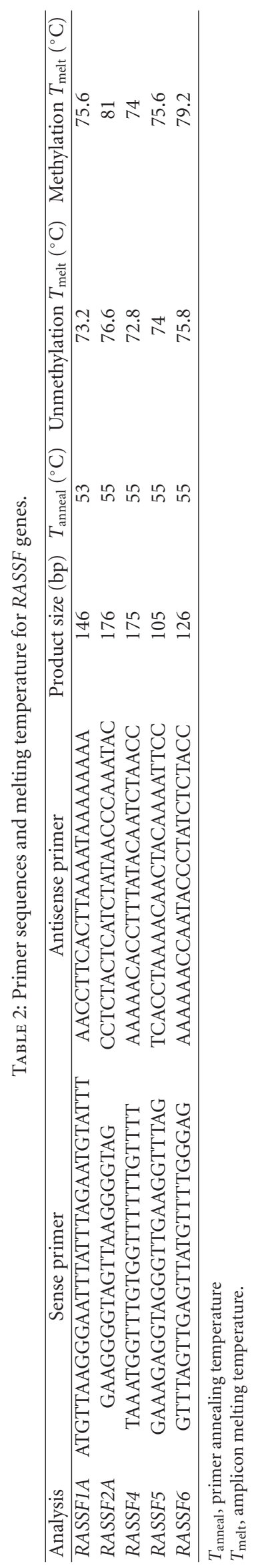




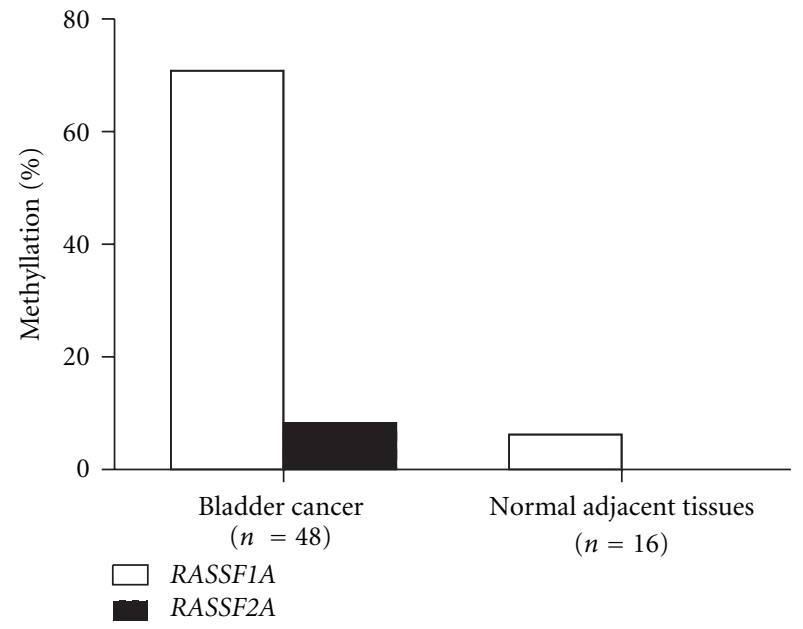

Figure 2: RASSF1A and RASSF2A methylation profiles of tumor and normal adjacent tissues. $71 \%(34 / 48)$ of tumor samples had RASSF1A promoter methylation, and $8 \%(4 / 48)$ had RASSF $2 A$ promoter methylation. No RASSF4, RASSF5, and RASSF6 promoter methylation were detected.

cases using MS-HRM [22]. 56\% (9/16) of tumor samples were found to have RASSF1A promoter methylation, and $25 \%(4 / 16)$ of tumor samples showed RASSF2A promoter methylation, while only $6 \%(1 / 16)$ of the normal adjacent tissue samples showed RASSF1A promoter methylation and none of normal adjacent tissue samples showed RASSF2A methylation. RASSF 4, 5, and 6 were not found to be methylated in either tumor or normal adjacent tissues. Figure 2 demonstrates that tumor and normal adjacent tissue showed different RASSF1A and RASSF2A methylation profiles.

Among the 48 patients with bladder cancer, RASSF1A promoter methylation alone had $71 \%$ sensitivity and $94 \%$ specificity and an area under the curve (AUC) of 0.823 to correctly identify bladder cancer tissue whereas RASSF1A and RASSF2A together had $77 \%$ sensitivity and $94 \%$ specificity and AUC of 0.854. RASSF1A and RASSF2A promoter methylation did discriminate bladder cancer tissue from normal adjacent tissue $(P<0.0001)$.

\subsection{RASSF1A and RASSF2A Methylation Profile in Different} Histological Samples. Patient characteristics are summarized in Table 1. 41 patients had tumors with transitional cell carcinoma features 4 with small cell carcinoma, and 3 with squamous cell carcinoma. RASSF1A was methylated in $68 \%$ $(28 / 41)$ and RASSF2A in 7\% (3/41) of transitional cell carcinoma samples. Only one sample had methylation of both the RASSF1A and RASSF2A promoters. RASSF1A was methylated in $100 \%(4 / 4)$ and RASSF $2 A$ in $0 \%(0 / 4)$ of small cell carcinoma. RASSF1A was methylated in $67 \%(2 / 3)$ and RASSF2A in $33 \%(1 / 3)$ of squamous cell carcinoma (Figure 3). The frequency of RASSF1 and RASSF2 promoter methylation together showed no significant difference with histology in our study $(P=0.295)$.

The reasons that lead to aberrant $\mathrm{CpG}$ island methylation of RASSF1A and RASSF2A in transitional cell carcinoma,

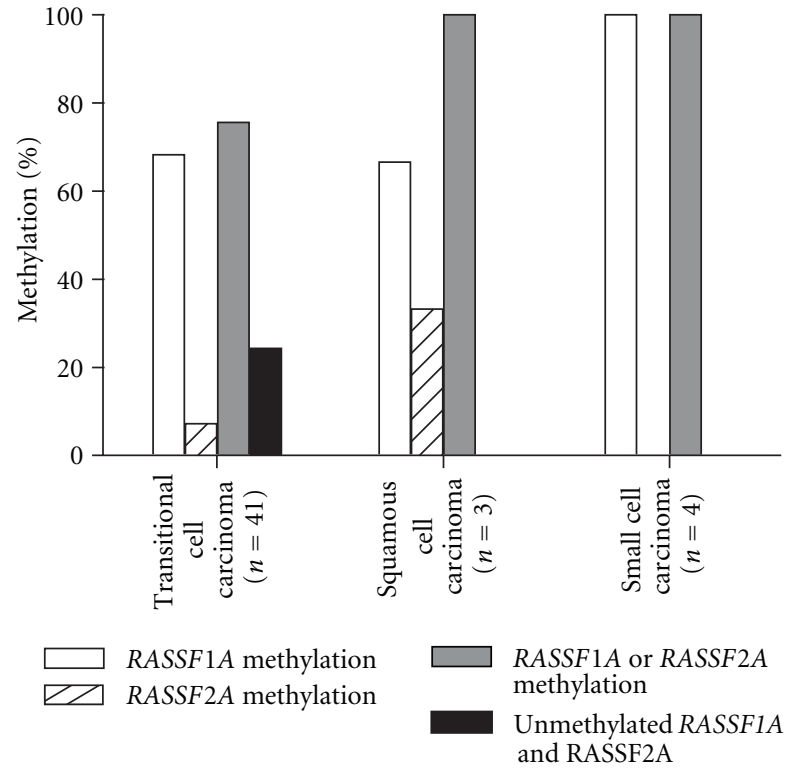

Figure 3: RASSF1A and RASSF2A methylation profiles of different histology. RASSF1A was methylated in 68\% (28/41) of transitional cell carcinoma samples, in $100 \%(4 / 4)$ of small cell carcinoma and in $67 \%(2 / 3)$ of squamous cell carcinoma. RASSF2A was methylated in $7 \%(3 / 41)$ of transitional cell carcinoma samples, in $0 \%(0 / 4)$ of small cell carcinoma, and in 33\% (1/3) of squamous cell carcinoma.

small cell carcinoma, and squamous cell carcinoma of the bladder are not well understood. Recent data by $\mathrm{Li}$ et al. investigating nonsmall cell lung cancer showed that RASSF1A promoter region CpG islands were methylated in $55 \%$ of adenocarcinomas, $25 \%$ of large cell carcinomas, and $25 \%$ of squamous cell carcinomas [3]. This study indicates that promoter methylation of RASSF gene family members might be dependent on histology in nonsmall cell lung cancer.

\subsection{RASSF1A and RASSF2A Methylation Profile at Different} $T$ Stages. 60 malignant bladder tumor and normal adjacent tissue samples from patients with different $\mathrm{T}$ stages (16 normal adjacent tissue samples, 9 stage $\mathrm{T} 1,11$ stage T2, 18 stage T3, and 6 stage T4) were analyzed to detect the RASSF promoter methylation changes of bladder cancer patients. Methylated RASSF1A promoters were only found in $6 \%(1 / 16)$ of normal adjacent tissues. The percentage of promoter methylation positive patients increased with $\mathrm{T}$ stage, being lower in $\mathrm{T} 1$ tumors and higher with higher stage. The percentage of samples with methylated RASSF1A genes was $55 \%(5 / 9)$ in stage $\mathrm{T} 1,73 \%(8 / 11)$ in stage $\mathrm{T} 2,78 \%$ $(14 / 18)$ in stage T3, and $83 \%(5 / 6)$ in specimens from T4 tumors (Figure 4). The frequency of RASSF1 and RASSF2 promoter methylation was not associated with $\mathrm{T}$ stage $(P=$ $0.363)$. Due to lack of samples with RASSF2A methylation, no association between RASSF2A methylation and $\mathrm{T}$ stage was identified in the current data set. Similar results were found in a recent lung cancer study [23]. The RASSF2A 


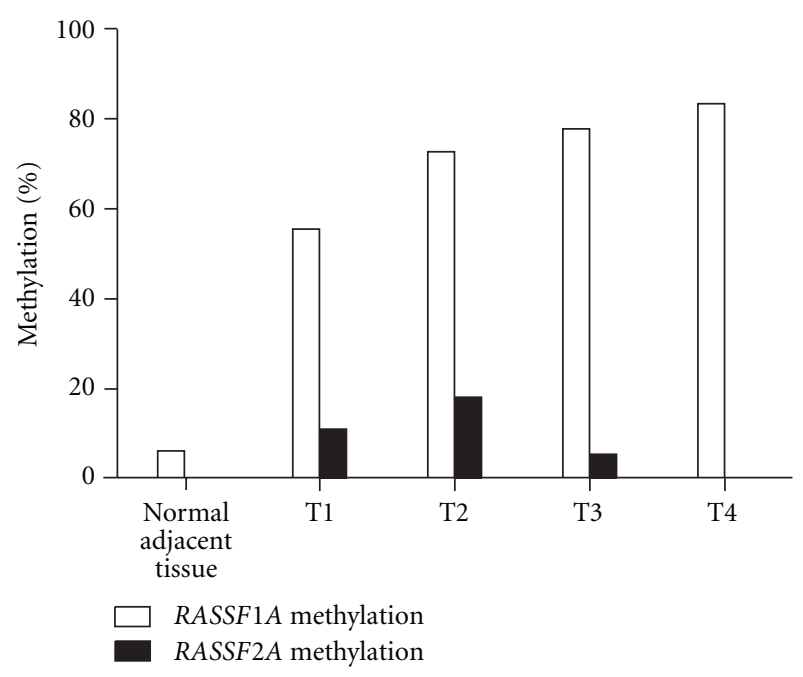

FIGURE 4: RASSF1A and RASSF2A methylation profiles in different $\mathrm{T}$ stage. RASSF1A was only $6 \%(1 / 16)$ in normal adjacent tissues, but percentage of tumor samples with methylated RASSF1A genes had a range of $55 \%-83 \%$ from $\mathrm{T} 1$ to $\mathrm{T} 4$ stage. The RASSF $2 A$ promoter had a low level (0-18\%) at different T stages.

promoter methylation was found at low levels (0-18\%) at different $\mathrm{T}$ stages.

\subsection{RASSF1A and RASSF2A Methylation Profile at Different} $N$ Stages. We examined methylation status of RASSF1A and RASSF2A in 27 bladder tumor samples with stage N0, 8 samples with stage $\mathrm{N} 1$, and 8 samples with stage $\mathrm{N} 2$. Percentage of RASSF1A promoter methylation had a range of $50-87.5 \%$ in tissues from patients with different lymph node metastasis stage. The percentage of samples with methylated RASSF2A was $11 \%(3 / 27)$ for stage N0, and $6 \%(1 / 16)$ for stage N1/2 (Figure 5). Based on the result of chi-square test, there is no significant difference between the frequency of RASSF1 and RASSF2 promoter methylation in lymph node positive and negative patients.

In nasopharyngeal carcinoma, aberrant methylation of $R A S S F 2 A$ promotor was found to be associated with lymph node metastasis [24]. Although both RASSF1A and RASSF2 protein can function as a negative effector of Ras protein in tumor formation, RASSF2A and RASSF1A have apparently different functions in different type of tumors.

3.6. RASSF4, RASSF5, and RASSF5 Methylation. RASSF1-6 share a variable $\mathrm{N}$-terminal sequence followed by a Ras-association domain [25]. The HRM analysis showed no detectable promoter methylation of RASSF4, RASSF5 and RASSF6 in bladder cancer and normal adjacent tissue samples.

\section{Discussion}

DNA methylation and histone modification are widely studied epigenetic events. Promoter hypermethylation has been proposed as a potential diagnostic or prognostic biomarker in various cancers. Recent research showed that urine is

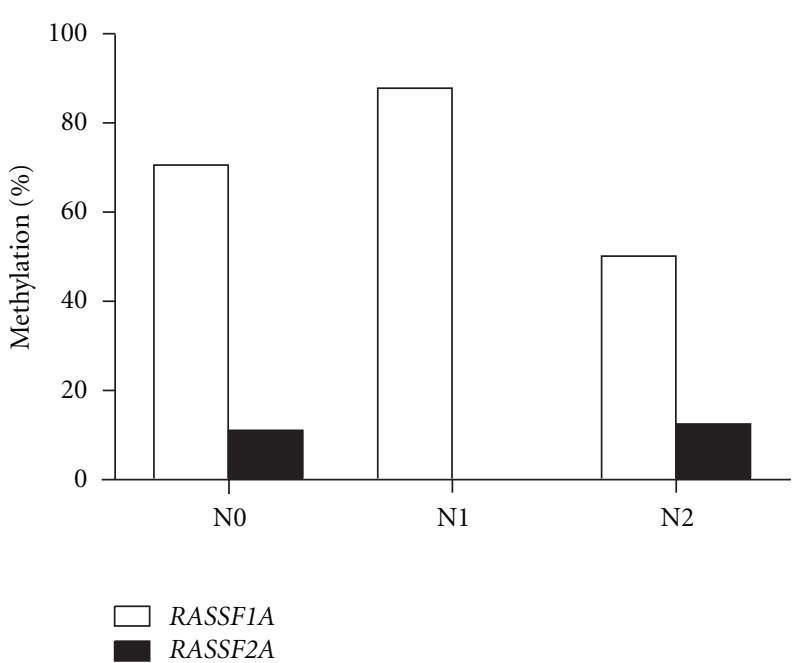

FIGURE 5: RASSF1A and RASSF2A methylation profiles in different $\mathrm{N}$ stages. Percentage of RASSF1A methylation had a range of 50$87.5 \%$ in different $\mathrm{N}$ stage, and percentage of RASSF $2 A$ had range of $0 \%-11 \%$. Aberrant methylation of RASSF1A and RASSF2A promotor showed no relationship to lymph node metastasis.

potentially useful for bladder cancer screening [26, 27]. Methylation status of certain genes identified in urine samples showed higher sensitivity than the conventional urine cytology method. These studies indicated that detection of promoter methylation in urine specimen could potentially provide a simple, noninvasive, and sufficiently sensitive method for bladder cancer screening in the future.

In our study, a new methodology, methylation-specifichigh resolution melting analysis was used to examine the melting behavior of methylated or unmethylated RASSF gene amplicons. This provides a simple and reproducible method for promoter methylation assessment. We studied DNA promoter methylation of five RASSF family members (RASSF1A, RASSF2A, RASSF4, RASSF5, and RASSF6) in FFPE bladder cancer tissues and normal adjacent tissues. We identified distinctive RASSF1A and RASSF2A gene promoter methylation profiles that differentiate between bladder cancer and normal adjacent tissue samples. Using RASSF1A and RASSF2A genes together showed an acceptable sensitivity (77\%) and high specificity (94\%) identifying bladder cancer tissues. Previous studies have identified RASSF1A promoter methylation as a potentially useful urine biomarker for the presence of invasive bladder cancer $[26,28$, 29]. We now show that the addition of RASSF $2 A$ promoter methylation analysis can improve the sensitivity potentially without compromising specificity. There was no significant correlation of RASSF1A methylation with histology and $\mathrm{N}$ stage. As discussed by Serizawa [1], results also showed that FGFR3 mutation in bladder cancer when combined with methylation markers (APC, RASSF1A and SFRP2) provided a sensitivity of $90 \%$ to identify bladder tumors. It remains to be shown if the addition of an RASSF $2 A$ promoter methylation assay to the previously published urine biomarker assay can indeed improve sensitivity when using urine samples. Limitations of the study include small sample size or lack 
of a validation data and sample set. In addition we did not have sufficient data to analyze if RASSF gene family promoter methylation could predict the recurrence of bladder cancer.

Methylation analysis of both RASSF1A and RASSF2A genes appeared to increase the sensitivity of discriminating cancer from normal adjacent tissue. The addition of RASSF2A methylation analysis to recent bladder cancer biomarker signatures has the potential to further increase sensitivity for bladder cancer diagnosis. RASSF1A and RASSF2A promoter methylation analysis could be useful as a biomarker to detect the presence of bladder cancer recurrence.

\section{Acknowledgments}

Tissue samples were provided by the Tissue Procurement Shared Resource at The Ohio State University Comprehensive Cancer Center, Columbus, Ohio. This paper is funded by the National Cancer Institute, Grant P30 CA16058. NIH/ NCI awards R01CA108633; RC2CA148190; the Brain Tumor Funders Collaborative Group; German Research Foundation DFG LA2510/1-1, and OSUCCC. Tissue samples were also provided by the Cooperative Human Tissue Network which is funded by the National Cancer Institute. Other investigators may have received specimens from the same subjects.

\section{References}

[1] R. R. Serizawa, U. Ralfkiær, K. Steven et al., "Integrated genetic and epigenetic analysis of bladder cancer reveals an additive diagnostic value of FGFR3 mutations and hypermethylation events," International Journal of Cancer, vol. 129, no. 1, pp. 7887, 2011.

[2] Y. K. Bae, A. Brown, E. Garrett et al., "Hypermethylation in histologically distinct classes of breast cancer," Clinical Cancer Research, vol. 10, no. 18 I, pp. 5998-6005, 2004.

[3] J. Li, Z. Zhang, Z. Dait et al., "RASSF1A promoter methylation and Kras2 mutations in non small cell lung cancer," Neoplasia, vol. 5, no. 4, pp. 362-366, 2003.

[4] Y. Wang, Z. Yu, T. Wang, J. Zhang, L. Hong, and L. Chen, "Identification of epigenetic aberrant promoter methylation of RASSF1A in serum DNA and its clinicopathological significance in lung cancer," Lung Cancer, vol. 56, no. 2, pp. 289-294, 2007.

[5] H. A. Ghazaleh, R. S. Chow, S. L. Choo et al., "14-3-3 mediated regulation of the tumor suppressor protein, RASSF1A," Apoptosis, vol. 15, no. 2, pp. 117-127, 2010.

[6] L. Shivakumar, J. Minna, T. Sakamaki, R. Pestell, and M. A. White, "The RASSF1A tumor suppressor blocks cell cycle progression and inhibits cyclin D1 accumulation," Molecular and Cellular Biology, vol. 22, no. 12, pp. 4309-4318, 2002.

[7] A. Dallol, A. Agathanggelou, S. L. Fenton et al., "RASSF1A interacts with microtubule-associated proteins and modulates microtubule dynamics," Cancer Research, vol. 64, no. 12, pp. 4112-4116, 2004.

[8] A. Agathanggelou, S. Honorio, D. P. Macartney et al., "Methylation associated inactivation of RASSF1A from region 3 p21.3 in lung, breast and ovarian tumours," Oncogene, vol. 20, no. 12, pp. 1509-1518, 2001.

[9] M. D. Vos, C. A. Ellis, C. Elam, A. S. Ülkü, B. J. Taylor, and G. J. Clark, "RASSF2 is a novel K-Ras-specific effector and potential tumor suppressor," Journal of Biological Chemistry, vol. 278, no. 30, pp. 28045-28051, 2003.

[10] L. Hesson, I. Bièche, D. Krex et al., "Frequent epigenetic inactivation of RASSF1A and BLU genes located within the critical 3p21.3 region in gliomas," Oncogene, vol. 23, no. 13, pp. 2408-2419, 2004.

[11] K. Eckfeld, L. Hesson, M. D. Vos, I. Bieche, F. Latif, and G. J. Clark, "RASSF4/AD037 is a potential Ras effector/tumor suppressor of the RASSF family," Cancer Research, vol. 64, no. 23, pp. 8688-8693, 2004.

[12] D. F. Calvisi, H. Donninger, M. D. Vos et al., "NORE1A tumor suppressor candidate modulates p21CIP1 via p53," Cancer Research, vol. 69, no. 11, pp. 4629-4637, 2009.

[13] J. Geli, P. Kogner, F. Lanner et al., "Assessment of NORE1A as a putative tumor suppressor in human neuroblastoma," International Journal of Cancer, vol. 123, no. 2, pp. 389-394, 2008.

[14] C. K. Lee, J. H. Lee, M. G. Lee et al., "Epigenetic inactivation of the NORE1 gene correlates with malignant progression of colorectal tumors," BMC Cancer, vol. 10, article 577, 2010.

[15] L. Buckingham, L. P. Faber, A. Kim et al., "PTEN, RASSF1 and DAPK site-specific hypermethylation and outcome in surgically treated stage I and II nonsmall cell lung cancer patients," International Journal of Cancer, vol. 126, no. 7, pp. 1630-1639, 2010.

[16] N. P. C. Allen, H. Donninger, M. D. Vos et al., "RASSF6 is a novel member of the RASSF family of tumor suppressors," Oncogene, vol. 26, no. 42, pp. 6203-6211, 2007.

[17] L. B. Hesson, T. L. Dunwell, W. N. Cooper et al., "The novel RASSF6 and RASSF10 candidate tumour suppressor genes are frequently epigenetically inactivated in childhood leukaemias," Molecular Cancer, vol. 8, article 42, 2009.

[18] T. K. Wojdacz, A. Dobrovic, and L. L. Hansen, "Methylationsensitive high-resolution melting," Nature protocols, vol. 3, no. 12, pp. 1903-1908, 2008.

[19] C. T. Wittwer, G. H. Reed, C. N. Gundry, J. G. Vandersteen, and R. J. Pryor, "High-resolution genotyping by amplicon melting analysis using LCGreen," Clinical Chemistry, vol. 49, no. 6, pp. 853-860, 2003.

[20] R. H. A. M. Vossen, E. Aten, A. Roos, and J. T. Den Dunnen, "High-resolution melting analysis (HRMA)—-more than just sequence variant screening," Human Mutation, vol. 30, no. 6, pp. 860-866, 2009.

[21] T. K. Wojdacz and A. Dobrovic, "Methylation-sensitive high resolution melting (MS-HRM): a new approach for sensitive and high-throughput assessment of methylation," Nucleic Acids Research, vol. 35, no. 6, article e41, 2007.

[22] I. Peters, K. Rehmet, N. Wilke et al., "RASSF1A promoter methylation and expression analysis in normal and neoplastic kidney indicates a role in early tumorigenesis," Molecular Cancer, vol. 6, article 49, 2007.

[23] S. Honorio, A. Agathanggelou, M. Schuermann et al., "Detection of RASSF1A aberrant promoter hypermethylation in sputum from chronic smokers and ductal carcinoma in situ from breast cancer patients," Oncogene, vol. 22, no. 1, pp. 147$150,2003$.

[24] Z. Zhang, D. Sun, N. V. Do, A. Tang, L. Hu, and G. Huang, "Inactivation of RASSF2A by promoter methylation correlates with lymph node metastasis in nasopharyngeal carcinoma," International Journal of Cancer, vol. 120, no. 1, pp. 32-38, 2007.

[25] L. van der Weyden and D. J. Adams, "The Ras-association domain family (RASSF) members and their role in human 
tumourigenesis," Biochimica et Biophysica Acta, vol. 1776, no. 1, pp. 58-85, 2007.

[26] M. W. Y. Chan, L. W. Chan, N. L. S. Tang et al., "Frequent hypermethylation of promoter region of RASSF1A in tumor tissues and voided urine of urinary bladder cancer patients," International Journal of Cancer, vol. 104, no. 5, pp. 611-616, 2003.

[27] J. Yu, T. Zhu, Z. Wang et al., "A novel set of DNA methylation markers in urine sediments for sensitive/specific detection of bladder cancer," Clinical Cancer Research, vol. 13, no. 24, pp. 7296-7304, 2007.

[28] T. C. Zuiverloon, W. Beukers, K. A. van der Keur et al., "A methylation assay for the detection of non-muscle-invasive bladder cancer (NMIBC) recurrences in voided urine," British Journal of Urology International, vol. 109, no. 6, pp. 941-948, 2012.

[29] C. J. Marsit, E. A. Houseman, B. C. Christensen et al., "Identification of methylated genes associated with aggressive bladder cancer," PLoS ONE, vol. 5, no. 8, article e12334, 2010. 

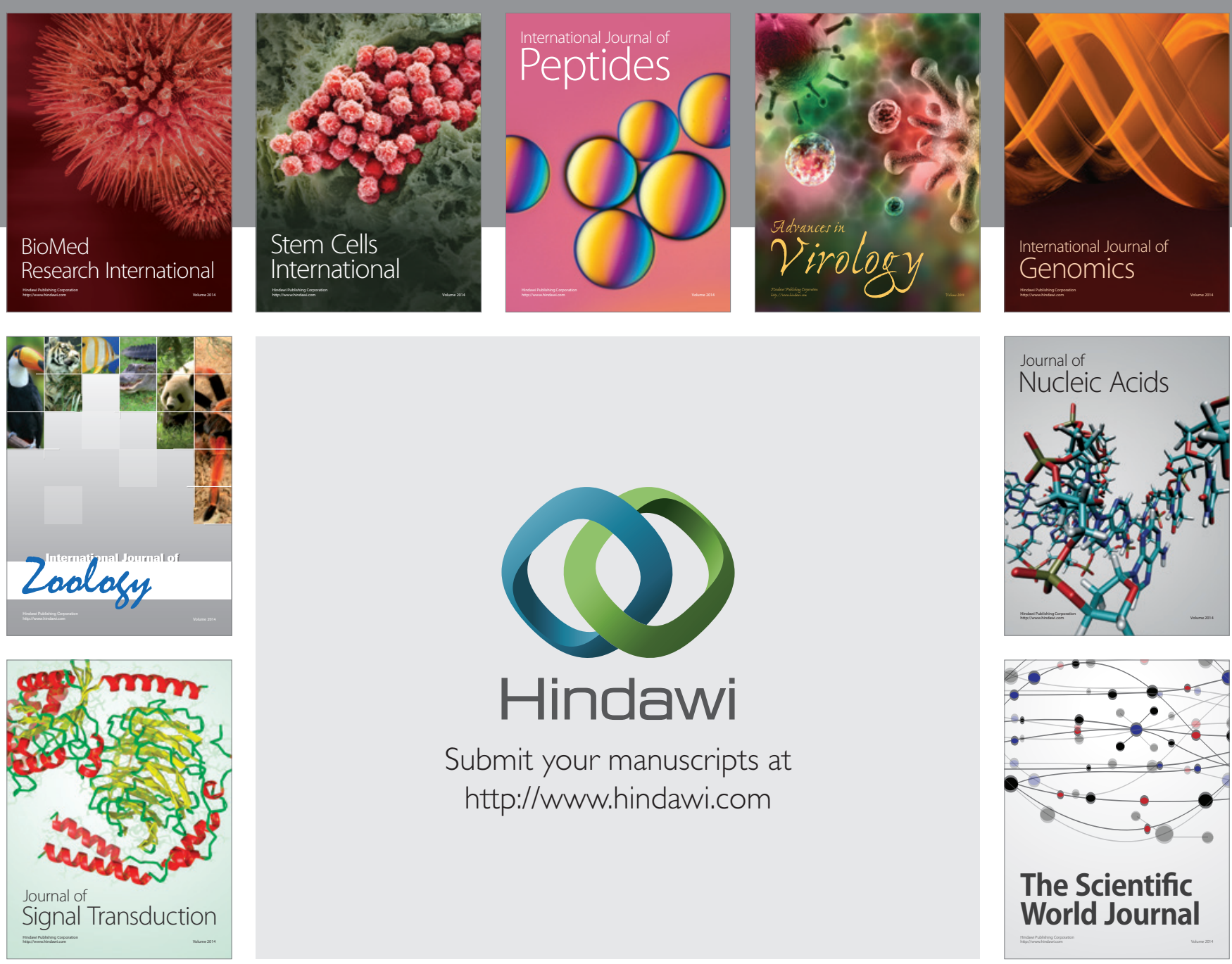

Submit your manuscripts at

http://www.hindawi.com
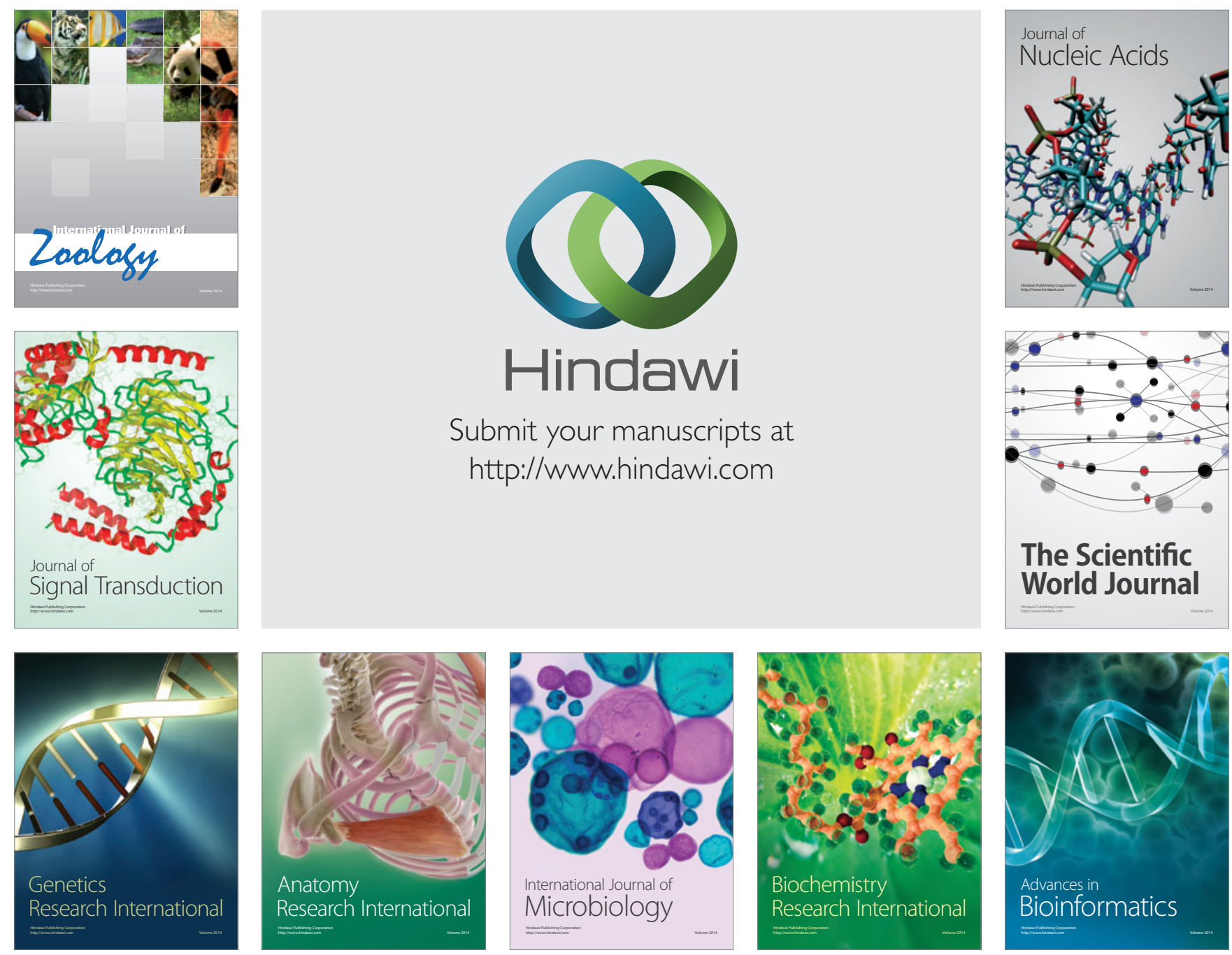

The Scientific World Journal
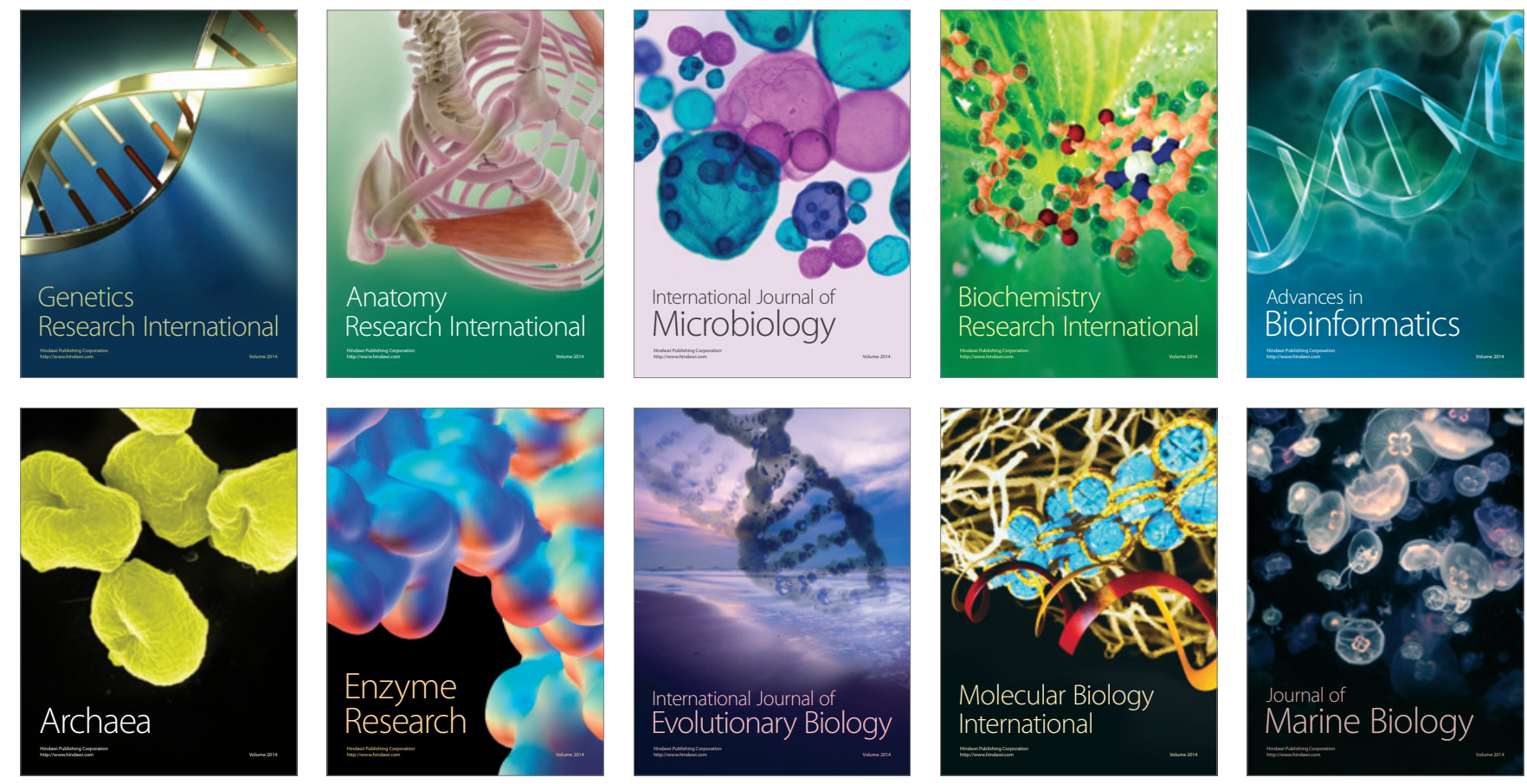